\title{
Energy spectrum of cascade showers initiated by cosmic ray muons in the Cherenkov water detector
}

\author{
S.S. Khokhlov ${ }^{1, *}$, A.G. Bogdanov ${ }^{1}$, V.A. Khomyakov ${ }^{1}$, V.V. Kindin ${ }^{1}$, R.P. Kokoulin ${ }^{1}$, A.A. Petrukhin ${ }^{1}$, V.V. Shutenko ${ }^{1}$, and \\ I.I. Yashin ${ }^{1}$
}

${ }^{1}$ National Research Nuclear University MEPhI (Moscow Engineering Physics Institute), 115409 Moscow, Russia

\begin{abstract}
Results of analysis of experimental data on cascade showers with energies of $0.1-10 \mathrm{TeV}$ initiated by muons in the Cherenkov water detector NEVOD are presented. The method of selection and reconstruction of cascade parameters in the volume of the detector is discussed. Experimental distributions of cascade axes in zenith angle and energy spectrum of cascades are obtained. The measured distributions are compared with results of calculations.
\end{abstract}

\section{Introduction}

Modern investigations of muons and neutrinos of ultrahigh energies are carried out using Cherenkov water detectors representing large water volumes of natural or artificial origin, in which optical sensors are deployed in the form of a regular spatial lattice.

One of the most important tasks of such detectors is to measure the energy of cascade showers generated by muons or neutrinos. Of particular interest is the measurement of the spectrum of cascades in the energy range 100 $\mathrm{GeV}-10 \mathrm{TeV}$, where a combined analysis of the zenith angular distribution and the energy spectrum of the cascades can provide information about the relative contribution of $K$ - and $\pi$-mesons to the generation of muons of cosmic rays.

However, in large-scale detectors the efficient reconstruction of cascades begins at energies of tens $\mathrm{TeV}$ due to a large distance between the optical modules. Therefore, the study of cascade showers in the Cherenkov water detector NEVOD with a dense spatial lattice of optical modules is an important experimental task.

The Cherenkov water detector of the experimental complex NEVOD [1] having a size of $9 \times 9 \times 26 \mathrm{~m}^{3}$ is equipped with a dense spatial lattice of 91 quasi-spherical modules (QSM). QSMs are placed with a step of $2.5 \mathrm{~m}$ along the detector, $2 \mathrm{~m}$ across it and $2 \mathrm{~m}$ in the vertical direction (Fig. 1). Each QSM has six FEU-200 PMTs with flat photocathodes which are directed along the axes of the orthogonal coordinate system. Such a design of the module allows to measure the intensity of Cherenkov radiation from relativistic charged particles with almost equal sensitivity for all directions of incident light; it also allows to determine the light direction. A density of the lattice of QSMs and a wide dynamic range of registered signals $\left(1-10^{5}\right.$ photoelectrons for each PMT $\left.[2,3]\right)$ allow

\footnotetext{
*e-mail: SSKhokhlov@mephi.ru
}

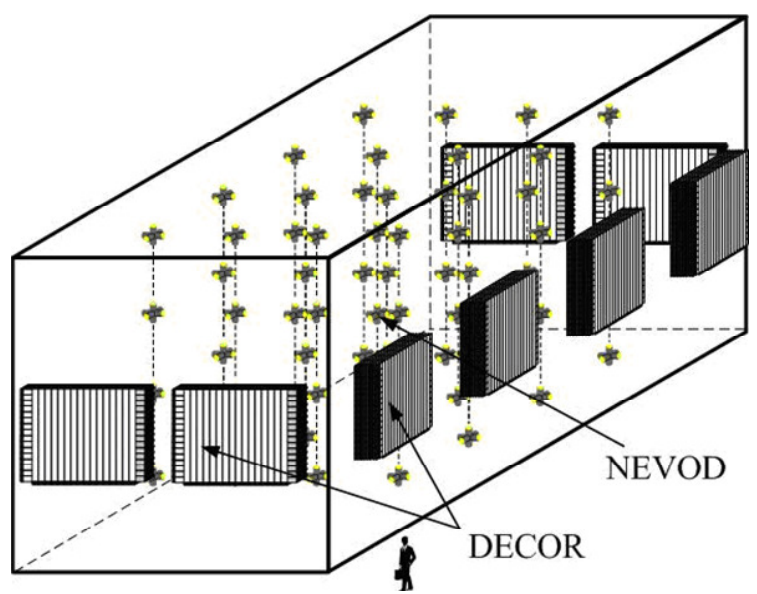

Figure 1. Experimental complex NEVOD-DECOR.

to measure the spatial distribution of Cherenkov radiation from cascade showers generated in the detector with about $0.5 \mathrm{~m}$ detalization.

These measurements were conducted for events with cascade showers generated by nearly horizontal muons in the sensitive volume of the detector. The muon track in each event was determined according to data of the coordinate-tracking detector DECOR [4]. Muons crossing the supermodules of the DECOR placed in the opposite galleries along two short sides of the Cherenkov detector were selected. Muons in such events have an average energy of $100 \mathrm{GeV}$ and zenith angles in the range of 85-90. The axis of the shower was assumed to coincide with the reconstructed muon track.

The reconstruction of parameters of cascade showers in these events was made by means of the method [5] using the assumption that all cascade particles move close to the axis of the shower and emit photons at the angle of about $42^{\circ}$. To measure the cascade curve of the shower, its axis 
was divided into bins of one radiation length each (about $36.1 \mathrm{~g} / \mathrm{cm}^{2}$ for water). The responses of PMTs that "see" the radiation from the axis bin at the Cherenkov angle were examined. The response of each such PMT was converted into the number of charged particles corresponding to the bin. In the case of several PMTs, the results were averaged taking into account the errors. The energy of each shower and its point of maximum were reconstructed by fitting the experimental cascade curve with one-dimensional analytical dependence of the number of particles on the depth [6].

\section{Spatial distribution of the Cherenkov radiation from cascades in water}

The spatial distribution of the Cherenkov radiation was measured for cascades with reconstructed energies of 100$500 \mathrm{GeV}$. 522 cascades (etalon sample) were selected among the data of experimental series 2013-2015 with 'live' time of about 12 thousand hours using the criteria described in the preceding section. The dependence of the QSM response on the distance from the axis and on the depth along the axis was measured. The response of the QSM was calculated as a square root of the sum of squared amplitudes of all its PMTs (this value does not practically depend on the direction of light [7]), normalized by the energy of the shower:

$$
B=\frac{\varepsilon_{0}}{\varepsilon} \sqrt{\sum A_{i}^{2}}
$$

where $\varepsilon_{0}=200 \mathrm{GeV}$ (the value close to the average energy of showers in the sample); $\varepsilon$ is the reconstructed energy of the shower in the event; $A_{i}$ is the amplitude of the signal of $i$-th PMT in the QSM.

The resulting dependence of $B$ on the depth and on the distance from the axis was calculated by averaging responses for all events in the sample. The zero point on the depth axis corresponds to the point of the maximum of each cascade.

Taking into account that the parameters of light attenuation in water are the same for cascade showers and for single muons, the ratio of light intensity $B$ measured for cascade showers to the intensity $B_{\mu}$ measured for single muons was considered. It allows to compensate the attenuation of Cherenkov light and to study more efficiently the angular distribution of cascade particles.

The longitudinal profiles of spatial distribution of $\left(B-B_{\mu}\right) / B_{\mu}$ value for different distances from the axis are presented in Fig. 2. The widening of the longitudinal profiles and decreasing of the maximum values with the distance from the axis are observed. This is a consequence of the divergence of Cherenkov light because of the scattering of cascade particles. Since the effect of light attenuation is mainly excluded, the areas under the curves of the profiles representing the energy of the shower are close to each other. In this case, the profile corresponding to the smallest distance from the shower axis (where the divergence of Cherenkov light is not significant) represents the average cascade curve.

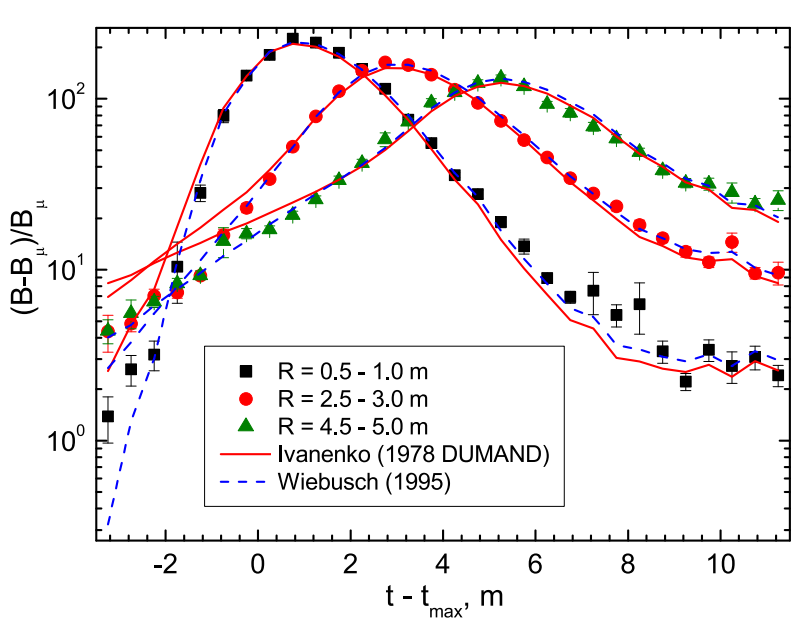

Figure 2. Longitudinal profiles of spatial distribution of the Cherenkov radiation from cascades.

The spatial distributions of the Cherenkov radiation for two models of angular distribution of cascade particles were calculated. First of these models was the analytical calculation on the basis of cascade equations performed by the group of I.P. Ivanenko [8]. The second one was the approximation (constructed by the IceCube collaboration [9]) of the results of modeling in Geant 3 by C. Wiebusch [10]. The results of comparison of experimental data with calculations are shown in Fig. 2, where the longitudinal profiles for three distances from the axis are presented.

Picture shows that in the region of maximum of the shower and at the decline of the dependences both models satisfactorily agree with the experiment. However, on the rising parts of the curves that correspond to the backward direction of light, the model of angular distribution of cascade electrons obtained by $\mathrm{C}$. Wiebusch is preferable.

\section{Selection of cascades and reconstruction of their parameters}

In the experiment, events with large energy deposits were selected by means of the trigger ' $60 \mathrm{c}$ '. A condition for this trigger was at least 60 QSMs were hit within a time gate of $250 \mathrm{~ns}$. Moreover, at least two PMTs in each of these modules should be hit.

In the NEVOD lattice, optical modules with the highest responses in the cascade events form a compact clusters. The geometry of the cluster can be used as a cascade signature (Fig. 3). It was found that the rms radius of the cluster of 20 QSM with the highest responses did not exceed $2.4 \mathrm{~m}$ in $95 \%$ of events of the etalon sample. The criterion of the compactness of the cluster of QSMs with highest responses was used to select cascade candidates.

The iterative algorithm for reconstructing the parameters of cascades is based on the method of least squares of the expected and experimental responses of individual PMTs. The calculation of the expected value of the response of the PMT is carried out taking into account the following models and assumptions. To determine the number of cascade particles from the depth of the shower 


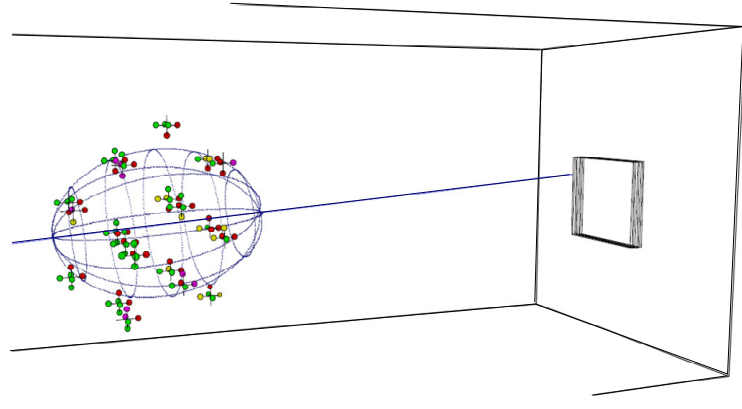

Figure 3. An example of the cluster of 20 QSMs with maximal responses.

development, the Greisen approximation for the cascade curve was used [6]. The angular distribution of Cherenkov radiation was determined on the basis of the $\mathrm{C}$. Wiebush model. As a response to a single particle, the experimental data on the PMT response in events with a single muon in NEVOD were used, taking into account the correction for the ratio of the average energy losses for muon and cascade electron in water.

The initial values of parameters of the reconstructed cascade were determined from the response of the QSM group with the largest responses: the cascade energy is proportional to the total response of such QSMs; the shower maximum is the center of mass of this group, estimated with the weights of their responses; the direction of the shower axis was estimated from the PMT responses taking into account their orientation in space.

The method of the reconstruction of the parameters of cascades was tested with cascade showers of the etalon sample and with cascades simulated in the Geant 4 toolkit [11]. The distributions of cascade showers in the spatial angle between the true and reconstructed axes are shown in Fig. 4 and Fig. 5. The use of individual photomultiplier responses in the reconstruction made it possible to achieve a good accuracy - the average spatial angle does not exceed $2.4^{\circ}$, and its standard deviation is $1.8^{\circ}$. The distribution of cascades in the ratio of the reconstructed and simulated cascade energy is presented in the Fig. 6. The accuracy of the reconstruction of shower energy is about $8 \%$.

\section{Energy spectrum of cascade showers}

The developed technique of selection of cascades and reconstruction of their parameters was applied to the data of the experimental series 2013-2017 (about 30 thousand hours of 'live' time). Fig. 7 shows zenith angular distributions of events with cascade showers for different energy ranges. At small zenith angles, the cascades generated by the hadronic component of cosmic rays dominate. For zenith angles above $55^{\circ}$ (the cosine of the angle is less than 0.57), the experimental data were fitted with the calculated curves of the zenith angular distributions of cascades generated exclusively by muons taking into account zenith angle measurement errors. A good overall agreement is observed.

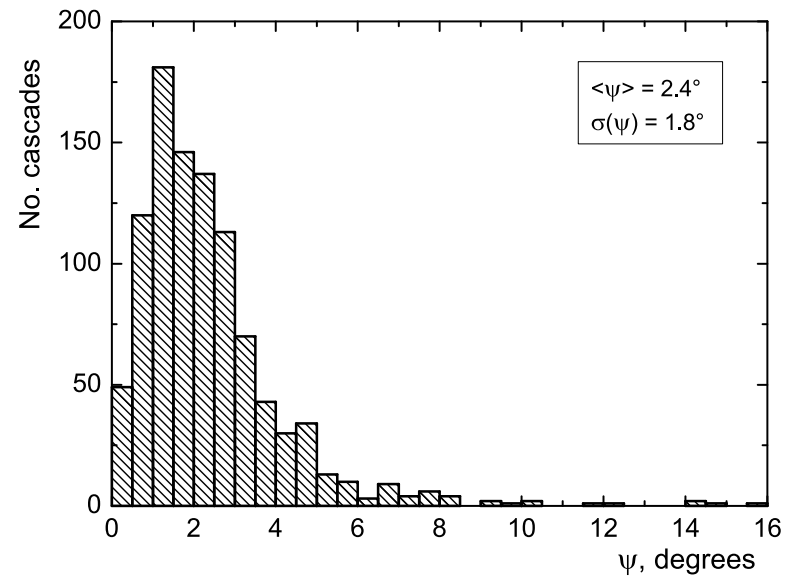

Figure 4. Distribution of cascades of the etalon sample in the spatial angle between the reconstructed cascade axes and the muon track measured by means of DECOR.

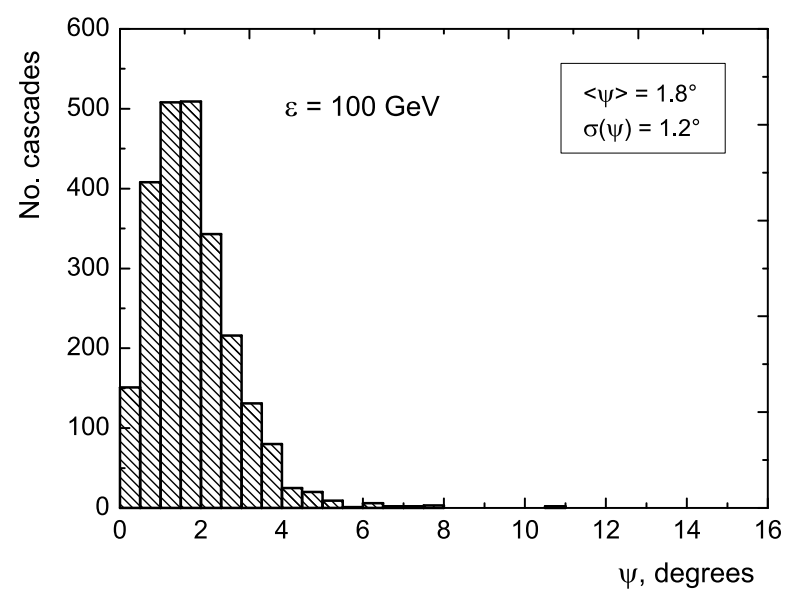

Figure 5. Distribution of cascades in the spatial angle between true and reconstructed axes for MC-events.

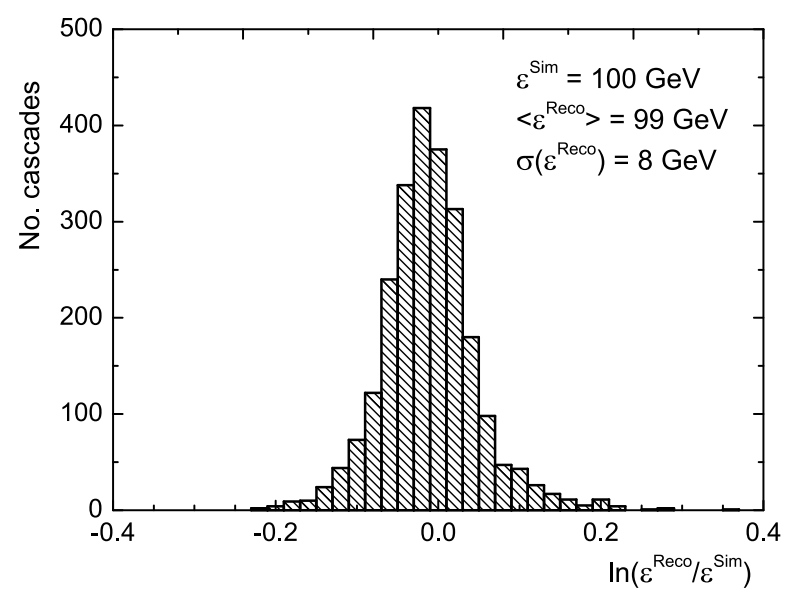

Figure 6. Distribution of cascades in the $\left(\varepsilon^{\mathrm{Reco}} / \varepsilon^{\mathrm{Sim}}\right)$-ratio.

The energy spectrum of cascades with zenith angles above $55^{\circ}$ is presented in Fig. 8. The results of calculations of expected spectra for different values of the slope 


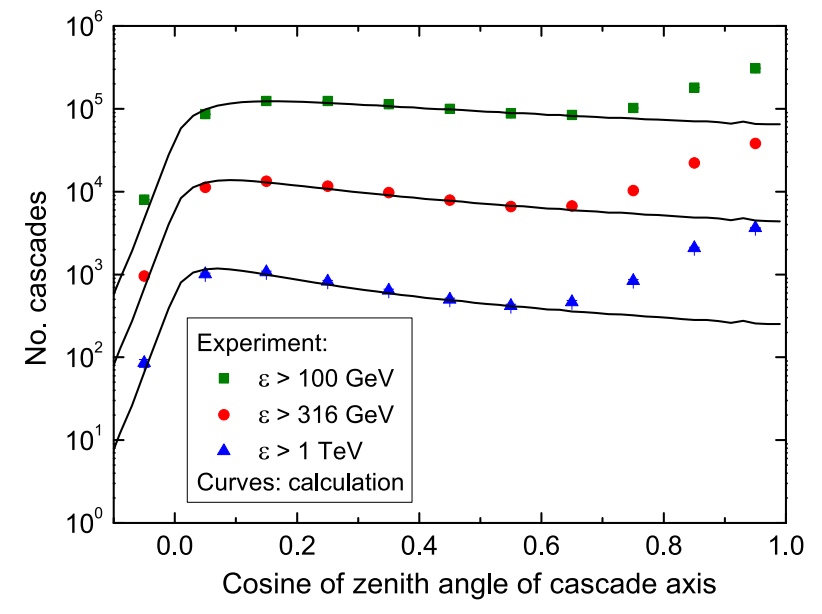

Figure 7. Zenith-angular distributions of cascades.

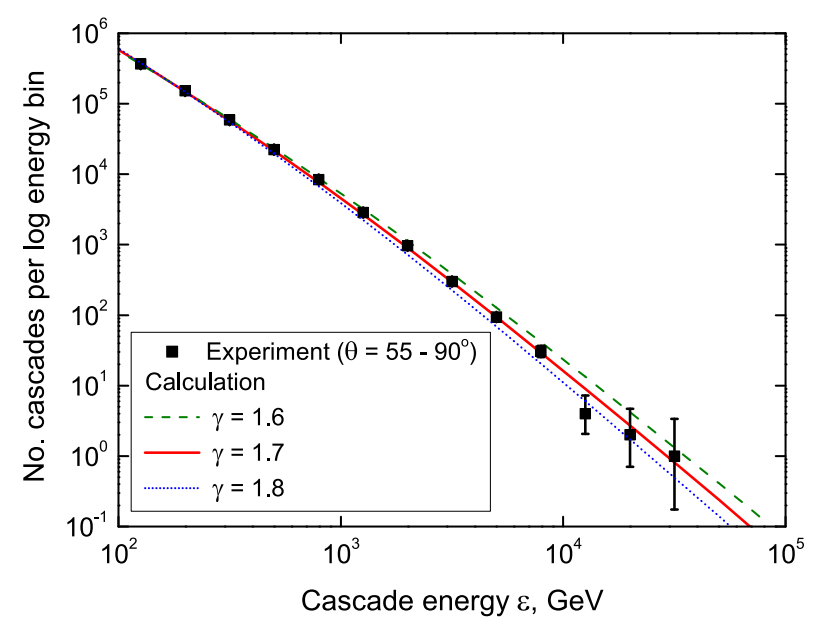

Figure 8. Energy spectrum of cascades initiated by muons with zenith angles exceeding $55^{\circ}$.

index of parent pion and kaon integral generation spectrum $\gamma$ are also shown. The likelyhood estimate of the in$\operatorname{dex}$ is $\gamma=1.65 \pm 0.01$. This optimization was carried out at a fixed ratio $K / \pi=0.15$. A further increase of statistics in future will allow to variate this parameter and obtain an experimental estimate of the relative contribution of $K$ mesons to the generation of muons.

\section{Conclusion}

A dense spatial lattice of quasispherical modules of the NEVOD detector has allowed for the first time to measure the spatial distribution of Cherenkov light from high energy cascade showers generated by muons. The comparison shows the agreement of experimental data with models describing cascade electron scattering.

The technique of reconstruction of cascades with an unknown axis has been developed and tested with the etalon experimental data sample and MC simulations. The differential energy spectrum of cascades generated by muons has been measured in the energy range of $\varepsilon=0.1-10 \mathrm{TeV}$, and the value of the index of pion and kaon integral generation spectrum was estimated as $\gamma=1.65 \pm 0.01$.

\section{Acknowledgments}

The work was performed at the Unique Scientific Facility "Experimental complex NEVOD" with the financial support provided by the Russian Ministry of Science and Higher Education (MEPhI Academic Excellence Project no. 02.a03.21.0005) and RFBR grant 15-02-07763-a. Simulations were performed using the resources of the $\mathrm{MEPhI}$ high-performance computing center.

\section{References}

[1] A.A. Petrukhin, Physics-Uspekhi 58, 486 (2015)

[2] V.V. Kindin et al., Physics Procedia 74, 435 (2015)

[3] V.V. Kindin et al., Instrum. Exp. Tech. 61, 649 (2018)

[4] N.S. Barbashina et al., Instrum. Exp. Tech. 43, 743 (2000)

[5] S.S. Khokhlov et al., Bull. Russ. Acad. Sci. Phys. 77, 638 (2013)

[6] S. Hayakawa, Cosmic Ray Physics (Wiley, Interscience, New York, 1969)

[7] V.A. Khomyakov et al., Physics Procedia 74, 442 (2015)

[8] A.A. Belayev et al., Proc. 1978 DUMAND Summer Workshop, Vol. 1, 337 (1978)

[9] M.G. Aartsen et al., NIM A 711, 73 (2013)

[10] C.H. Wiebusch, The detection of faint light in deep underwater neutrino telescopes $(\mathrm{Ph} . \mathrm{D}$. Thesis, Physikalische Institute RWTH Aachen, 1995)

[11] J. Allison et al., NIM A 835, 186 (2016) 